\title{
Prevalence of and factors associated with anxiety and depression in Korean patients with newly diagnosed advanced gastrointestinal cancer
}

Junghwa Chung ${ }^{1}$, Gawon Ju ${ }^{2}$, Jiyoul Yang ${ }^{1}$, Jiwon Jeong ${ }^{1}$, Yusook Jeong ${ }^{1}$, Moon Ki Choi ${ }^{1}$, Jihyun Kwon ${ }^{1}$, Ki Hyeong Lee ${ }^{1,3}$, Seung Taik Kim ${ }^{1,3}$, and Hye Sook Han ${ }^{1,3}$

Departments of ${ }^{1}$ Internal Medicine and ${ }^{2}$ Psychiatry, Chungbuk National University Hospital, Cheongju; ${ }^{3}$ Department of Internal Medicine, Chungbuk National University College of Medicine, Cheongju, Korea

\author{
Received: March 25, 2016 \\ Revised : August 21, 2016 \\ Accepted: October 7, 2016 \\ Correspondence to \\ Hye Sook Han, M.D. \\ Department of Internal \\ Medicine, Chungbuk National \\ University College of Medicine, \\ 1 Chungdae-ro, Seowon-gu, \\ Cheongju 28644, Korea \\ Tel: $+82-43-269-6306$ \\ Fax: $+82-43-273-3252$ \\ E-mail: sook3529@hanmail.net
}

Background/Aims: The purpose of the present study was to assess the prevalence of and factors associated with anxiety and depression in Korean patients with advanced gastrointestinal cancer.

Methods: One hundred and twenty consecutive patients with newly diagnosed, advanced gastrointestinal cancer who were scheduled to receive palliative chemotherapy between July 2012 and June 2014 were enrolled in this observational prospective study. Anxiety and depression were assessed using the Hospital Anxiety and Depression Scale (HADS) and Patient Health Questionnaire-9 (PHQ-9).

Results: Thirty-seven patients (30.8\%) had anxiety or depression with clinical significance according to HADS or PHQ-9. Multivariate analysis identified lower performance status (odds ratio [OR], 4.19; 95\% confidence interval [CI], 1.22 to $14.35 ; p=0.023$ ), gastric cancer (OR, 5.39; $95 \% \mathrm{CI}, 0.37$ to $78.23 ; p=0.018)$, and knowledge of advanced cancer (OR, 15.07; 95\% CI, 1.80 to 125.90; $p=0.012$ ) as significantly associated with anxiety or depression. Twenty-one patients with anxiety or depression visited the psycho-oncologic clinic. In these patients, PHQ-9 score ( $p$ $=0.008)$, global health status $(p=0.023)$, fatigue $(p=0.047)$, and appetite loss $(p=$ 0.006) improved from baseline to 3 months after study enrollment.

Conclusions: Approximately $30 \%$ of Korean patients with advanced gastrointestinal cancer had anxiety or depression. The prevalence of anxiety or depression was higher in patients with poor performance status, gastric cancer, or knowledge of advanced cancer. Psychiatric interventions may be effective in reducing depression and improving quality of life in cancer patients with anxiety or depression.

Keywords: Anxiety; Neoplasms; Depression; Gastrointestinal

\section{INTRODUCTION}

Distress in cancer patients is a multifactorial, unpleasant emotional experience of a psychological, social, and/ or spiritual nature that may interfere with the ability to cope effectively with cancer, its physical symptoms, and its treatment [1]. For this reason, distress is referred to as the sixth vital sign in cancer care and should be recognized, monitored, documented, and treated promptly at all stages of disease and in all settings [1]. Psychological distress, such as anxiety or depression, in cancer patients reduces quality of life (QOL), negatively impacts compliance with medical treatment, and carries an elevated risk of mortality [2-6]. A recent Korean study re- 
ported that the prevalence of psychological distress was $56.5 \%$ in cancer patients, and that psychological distress was associated with poor performance status [7]. Another Korean study reported that $28.8 \%$ of cancer patients experienced psychological distress, and female gender, low education level, and low performance status were significantly associated with this condition [8].

Anxiety and depression are more common in advanced cancer than in the early stage of cancer, where the prevalence is $25 \%$ to $65 \%$ and $22 \%$ to $56 \%$, respectively [7-13]. The wide variation in reported prevalence can be attributed to different research samples with different risk factors, measurement tools, and diagnostic criteria. Although there are many studies on psychological distress in cancer patients, most studies on anxiety and depression have focused on all cancers, or on breast or lung cancers, and there are few studies on the prevalence of and factors associated with psychological distress in patients with gastrointestinal cancer [14-16]. Furthermore, although the incidence of gastrointestinal cancer in Korea is relatively high compared with other cancers [17], there are no studies on the prevalence of and factors associated with anxiety or depression in Korean patients with advanced gastrointestinal cancer.

The objective of this study was to assess the prevalence of and factors associated with anxiety and depression in Korean patients with advanced gastrointestinal cancer requiring palliative chemotherapy, and to investigate the effect of psychiatric intervention in patients with anxiety or depression.

\section{METHODS}

\section{Patients}

All eligible patients with newly diagnosed advanced or metastatic gastrointestinal cancer in Chungbuk National University Hospital were considered for this study. The following inclusion criteria were applied: age $>18$ years; histologically confirmed advanced gastrointestinal cancer including esophagus, gastric, colorectal, liver, pancreas, and bile duct cancer; scheduled to receive palliative chemotherapy; life expectancy $\geq 3$ months; ability to understand the Korean language; and normal cognitive function. Patients were excluded if they had a terminal condition, low performance status (Eastern
Cooperative Oncology Group [ECOG] performance status $\geq 3$ ), cognitive impairment, history of a psychological disorder or substance abuse, or a severe comorbid illness. Written informed consent was obtained from all participants prior to enrollment in the study. This observational study was reviewed and approved by the Institutional Review Board of Chungbuk National University Hospital.

\section{Procedure}

Anxiety, depression, and QOL were evaluated at initial diagnosis of advanced gastrointestinal cancer (baseline) and at 3 months after study enrollment. Patients with anxiety or depression at baseline were invited to visit a psycho-oncology clinic, and patients who agreed to visit the psycho-oncology clinic were invited for a psychiatric assessment. Major psychiatric disorders were evaluated face-to-face by one psychiatrist using the Mini International Neuropsychiatric Interview, Korean version 5.0 [18]. A psychiatric diagnosis was made according to the criteria of the fourth edition of the Diagnostic and Statistical Manual of Mental Disorders (DSM-IV) [19].

\section{Sociodemographic and clinical characteristics}

Sociodemographic data (marital status, education level, employment status, monthly income, and knowledge of advanced cancer that could only be treated with palliative therapy) were obtained from standardized questions. Clinical data (age, sex, ECOG performance status, and type of cancer) were obtained from a physician's assessment and the patient's medical record.

\section{Patient-reported measures}

Anxiety and depression were evaluated using both the Hospital Anxiety and Depression Scale (HADS) and the Patient Health Questionnaire-9 (PHQ-9), Korean version. QOL was evaluated using the European Organization for Research and Treatment of Cancer Quality of Life Questionnaire Core 30 (EORTC QLQ-C30), Korean version 3 .

The HADS is a 14-item questionnaire consisting of two subscales, anxiety and depression, each including seven items. Each item is scored on a four-point scale ranging from zero to three, and subscale scores range from zero, indicating no distress, to 21, indicating maximum distress. A score $\geq 8$ on either of the two HADS 
subscales is considered to be clinically significant [20]. The PHQ- 9 is a nine-item measure that evaluates symptoms of major depressive disorder according to the criteria of the DSM-IV. Each item is rated on a scale of zero to three and the total score ranges from zero to 27 . A major depressive disorder was considered if a patient reported at least five of the nine symptoms of depression on the PHQ-9, with one of the five symptoms being either depressed mood or anhedonia, and a high score indicated a high level of depression [21].

The EORTC QLQ-C30 is a cancer-specific questionnaire that evaluates health-related QOL. It consists of 30 items and is organized into the following dimensions: global health status, function (physical function, role function, cognitive function, emotional function, and social function), and symptoms (fatigue, nausea and vomiting, pain, dyspnea, insomnia, loss of appetite, constipation, diarrhea, and financial difficulties). Higher scores for global health status and on the functional scales indicate better overall QOL and level of functioning, and higher scores on the symptom scales indicate a higher level of symptomatology and worse QOL [22].

\section{Statistical analysis}

Data are summarized as frequencies and percentages for categorical variables, and mean \pm standard deviation for continuous variables. Chi-square tests were used to compare percentages, and independent sample $t$ tests were used to compare mean values between patients with and without anxiety or depression. Univariate and multivariate logistic regression analyses were performed to investigate the relations between anxiety or depression and sociodemographic or clinical variables. Sociodemographic and clinical variables associated with anxiety or depression in univariate analyses $(p<0.25)$ were entered into a multivariate logistic regression analysis. A paired $t$ test was used to compare HADS score, PHQ-9 score, and score on each dimension of the EORTC QLQ-C3O between baseline and 3 months after study enrollment for patients without anxiety or depression at baseline, patients with anxiety or depression at baseline who did not visit the psycho-oncology clinic, and patients with anxiety or depression at baseline who did visit the psycho-oncology clinic. A $p<0.05$ was considered statistically significant. All statistical analyses were performed using SPSS version 18.0 (SPSS Inc., Chicago, IL, USA).

\section{RESULTS}

\section{Patient characteristics}

Of the 129 patients screened between July 2012 and June 2014, 120 were included in the study. Nine patients were excluded because of cognitive impairment $(n=2)$, refusal to participate $(n=4)$, or inability to fill in the questionnaires $(n=3)$ (Fig. 1$)$. Therefore, the response rate was $93.0 \%$ during the study period. Of the 120 patients included in the study, 100 patients completed the anxiety, depression, and QOL evaluations at baseline and at 3 months after study enrollment, and 20 had incomplete data at 3 months because they were lost to follow-up (n $=11$ ) or died before the 3 months assessment $(n=9)$ (Fig. 1). The sociodemographic and clinical characteristics of the 120 patients included in the study are shown in Table 1. The median age of the patients was 63.0 years (range, 34 to 88). The majority of patients were male (76.7\%), married (76.7\%), unemployed (76.7\%), and had good ECOG performance status (ECOG performance status $\leq 1,86.7 \%)$. The primary sites of tumor were esophagus (4.2\%), stomach (26.7\%), colorectum (33.3\%), and liver or pancreas or biliary tract (35.8\%). Ninety patients (75.0\%) knew they had advanced cancer that could only be treated with palliative therapy, and 30 patients (25.0\%) did not know.

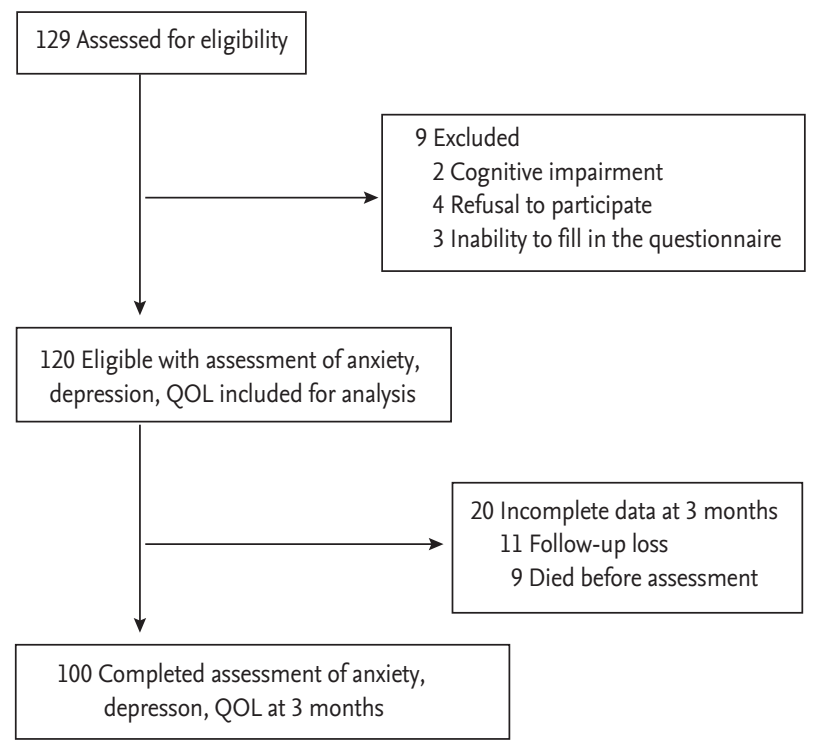

Figure 1. Flow diagram showing patient selection. QOL, quality of life. 
Table 1. Sociodemographic and clinical characteristics

\begin{tabular}{|c|c|}
\hline Characteristic & No. of patients $(n=120)$ \\
\hline \multicolumn{2}{|l|}{ Age, yr } \\
\hline$\leq 65$ & $69(57.5)$ \\
\hline$>65$ & $5^{1}(42.5)$ \\
\hline \multicolumn{2}{|l|}{ Sex } \\
\hline Male & $92(76.7)$ \\
\hline Female & $28(23 \cdot 3)$ \\
\hline \multicolumn{2}{|l|}{ ECOG performance status } \\
\hline o & $13(10.8)$ \\
\hline 1 & $91(75 \cdot 9)$ \\
\hline 2 & $16(13 \cdot 3)$ \\
\hline \multicolumn{2}{|l|}{ Primary site of tumor } \\
\hline Esophagus & $5(4 \cdot 2)$ \\
\hline Stomach & $32(26.7)$ \\
\hline Colorectum & $40(33 \cdot 3)$ \\
\hline Liver, pancreas, or bile duct & $43(35.8)$ \\
\hline \multicolumn{2}{|l|}{ Marital status } \\
\hline Married & $92(76.7)$ \\
\hline Not married & $28(23 \cdot 3)$ \\
\hline \multicolumn{2}{|l|}{ Education level } \\
\hline$\leq$ Middle school & $78(65.0)$ \\
\hline$\geq$ High school & $42(35 \cdot 0)$ \\
\hline \multicolumn{2}{|l|}{ Employment status } \\
\hline Employed & $28(23.3)$ \\
\hline Unemployed & $92(76.7)$ \\
\hline \multicolumn{2}{|l|}{ Monthly income, won } \\
\hline$\leq 1,000,000$ & $74(61.7)$ \\
\hline$>1,000,000$ & $46(38.3)$ \\
\hline \multicolumn{2}{|l|}{ Knowledge of advanced cancer } \\
\hline Yes & $90(75.0)$ \\
\hline No & $30(25.0)$ \\
\hline
\end{tabular}

Values are presented as number (\%).

ECOG, Eastern Cooperative Oncology Group.

\section{Prevalence of and factors associated with anxiety or depression}

The HADS score at baseline was $2.60 \pm 2.97$ for the anxiety subscale and $5.49 \pm 3.29$ for the depression subscale, and the PHQ-9 score at baseline was $6.40 \pm 4.60$. Of the 120 patients, 11 (9.2\%) had anxiety and $32(26.7 \%)$ had depression according to the HADS, and 31 (25.8\%) met the diagnostic criteria for major depressive episode according to the PHQ-9(Table 2). Overall, 37 of the 120 patients
Table 2. The prevalence of anxiety and depression as determined by the HADS and the PHQ-9

\begin{tabular}{lc}
\hline Variable & No. of patients $(\mathrm{n}=120)$ \\
\hline HADS-A & $11(9.2)$ \\
HADS-D & $32(26.7)$ \\
PHQ-9 & $31(25.8)$ \\
Any & $37(30.8)$ \\
\hline
\end{tabular}

Values are presented as number (\%).

HADS-A, Hospital Anxiety and Depression Scale-Anxiety; HADS-D, Hospital Anxiety and Depression Scale-Depression; PHQ-9, Patient Health Questionnaire-9.

(30.8\%) were identified as having anxiety or depression according to HADS or PHQ-9. Of these 37 patients with anxiety or depression, 28 (75.7\%) received a psychiatric assessment in the psycho-oncology clinic, and psychiatric diagnoses were made. The diagnoses were adjustment disorder $(\mathrm{n}=11,39.3 \%)$, major depressive disorder ( $\mathrm{n}=12,42.8 \%$ ), and depressive disorder not otherwise specified $(n=1,3.6 \%)$. Four patients $(14.3 \%)$ did not receive any psychiatric diagnosis.

In univariate analysis, the prevalence of anxiety or depression was significantly affected by age, ECOG performance status, and knowledge of advanced cancer (Table 3). The prevalence of anxiety or depression in patients aged $\leq 65$ years was greater than that in patients aged $>65$ years (39.1\% vs. 19.6\%, $p=0.037$ ). The prevalence of anxiety or depression increased as ECOG performance status decreased (0\%, 30.8\%, and 56.3\% in patients with ECOG performance status of $\mathrm{o}, 1$, and 2, respectively; $p$ $=0.005)$. The prevalence of anxiety or depression was greater in patients who knew they had advanced cancer than in patients who did not know they had advanced cancer (40.0\% vs. $3.3 \%, p<0.001)$.

Multivariate analysis revealed that lower performance status (odds ratio [OR], 4.19; 95\% confidence interval [CI], 1.22 to $14.35 ; p=0.023)$, gastric cancer (OR, 5.39; 95\% CI, 0.37 to $78.23 ; p=0.018$ ), and knowledge of advanced cancer (OR, 15.07; 95\% CI, 1.80 to 125.90; $p=0.012$ ) were significantly associated with anxiety or depression (Table 3). Age, sex, marital status, education level, employment status, and monthly income were not significantly associated with anxiety or depression (Table 3). 
Table 3. Univariate and multivariate analysis of sociodemographic and clinical characteristics associated with anxiety or depression

\begin{tabular}{|c|c|c|c|c|c|}
\hline \multirow[b]{2}{*}{ Characteristic } & \multicolumn{3}{|c|}{ Univariate analysis } & \multicolumn{2}{|c|}{ Multivariate analysis } \\
\hline & $\begin{array}{l}\text { Without anxiety or } \\
\text { depression }(n=83)\end{array}$ & $\begin{array}{c}\text { With anxiety or } \\
\text { depression }(n=37)\end{array}$ & $p$ value & OR $(95 \% \mathrm{CI})$ & $p$ value \\
\hline Age, yr & & & & & 0.108 \\
\hline$\leq 65(n=69)$ & $42(60.9)$ & $27(39.1)$ & 0.037 & $2.78(0.80-9.67)$ & \\
\hline$>65(\mathrm{n}=51)$ & $41(80.4)$ & $10(19.6)$ & & & \\
\hline Sex & & & 0.950 & & \\
\hline Male $(n=92)$ & $63(68.5)$ & $29(31.5)$ & & & \\
\hline Female $(\mathrm{n}=28)$ & $20(71.4)$ & $8(28.6)$ & & & \\
\hline ECOG performance status & & & 0.005 & & 0.023 \\
\hline$o(n=13)$ & $13(100.0)$ & o & & & \\
\hline $1(\mathrm{n}=91)$ & $63(69.2)$ & $28(30.8)$ & & & \\
\hline $2(n=16)$ & $7(43.8)$ & $9(56.3)$ & & $4.19(1.22-14.35)$ & \\
\hline Type of cancer & & & 0.106 & & 0.018 \\
\hline Esophagus $(\mathrm{n}=5)$ & $4(80.0)$ & $1(20.0)$ & & & \\
\hline Stomach $(\mathrm{n}=32)$ & $17(53.1)$ & $15(46.9)$ & & $5.39(0.37-78.23)$ & \\
\hline Colorectum $(n=40)$ & $28(70.0)$ & $12(30.0)$ & & $3.63(0.25-52.81)$ & \\
\hline Liver, pancreas, or bile duct $(n=43)$ & $34(79.1)$ & $9(20.9)$ & & $1.43(0.10-20.95)$ & \\
\hline Marital status & & & 0.596 & & \\
\hline Married $(n=92)$ & $62(67.4)$ & $30(32.6)$ & & & \\
\hline Not married $(n=28)$ & $21(75 \cdot 0)$ & $7(25 \cdot 0)$ & & & \\
\hline Education level & & & 0.141 & & 0.934 \\
\hline$\leq$ Middle school $(\mathrm{n}=78)$ & $58(74 \cdot 4)$ & $20(25.6)$ & & & \\
\hline$\geq$ High school $(n=42)$ & $25(59 \cdot 5)$ & $17(40.5)$ & & $1.04(0.38-2.89)$ & \\
\hline Employment status & & & 0.319 & & \\
\hline Employed $(\mathrm{n}=28)$ & $22(78.6)$ & $6(21.4)$ & & & \\
\hline Unemployed $(\mathrm{n}=92)$ & $61(66.3)$ & $31(33.7)$ & & & \\
\hline Monthly income, won & & & 0.079 & & 0.067 \\
\hline$\leq 1,000,000(\mathrm{n}=74)$ & $56(75 \cdot 7)$ & $18(24 \cdot 3)$ & & & \\
\hline$>1,000,000(n=46)$ & $27(58.7)$ & $19(41.3)$ & & $2.67(0.93-7.63)$ & \\
\hline Knowledge of advanced cancer & & & $<0.001$ & & 0.012 \\
\hline Yes $(n=90)$ & $54(60.0)$ & $36(40.0)$ & & $15.07(1.80-125.90)$ & \\
\hline No $(\mathrm{n}=30)$ & $29(96.7)$ & $1(3 \cdot 3)$ & & & \\
\hline
\end{tabular}

Values are presented as number (\%).

OR, odds ratio; CI, confidence interval; ECOG, Eastern Cooperative Oncology Group.

\section{Change in anxiety, depression, and QOL from base-} line to 3 months

Of the 120 patients included in the study, 100 (83.3\%) completed the assessment at 3 months after study enrollment. This corresponds to 71 of the 83 patients (85.6\%) without anxiety or depression at baseline and 29 of the 37 patients (78.4\%) with anxiety or depression at baseline. Of the 29 patients with anxiety or depression, 21 (72.4\%) underwent a psychiatric assessment and a psychiatric intervention in the psycho-oncology clinic. The psychiatric interventions included psychotherapy $(\mathrm{n}=7$, $33.3 \%)$ and psychiatric medications $(n=14,66.7 \%)$ such 
as antidepressants, antianxiety drugs, and sleeping pills.

In the 71 patients without anxiety or depression at baseline, HADS anxiety score $(p=0.606)$ and PHQ-9 score $(p=0.217)$ did not change between baseline and 3 months, but there was a significant increment in HADS depression score $(p=0.009$ ) (Table 4 , Fig. 2A). In the eight patients with anxiety or depression who did not visit the psycho-oncologic clinic, there was no change in anxiety or depression from baseline to 3 months (Table 4, Fig. $2 \mathrm{~B})$. However, in the 21 patients with anxiety or depression who did visit the psycho-oncologic clinic, there was a significant decrement in PHQ-9 score $(p=0.008)$ (Table 4, Fig. $2 \mathrm{C}$ ), indicating an improvement in depressive symptoms, and an improvement in global health status $(p=0.023)$, fatigue $(p=0.047)$, and appetite loss $(p=0.006)$ at 3 months after study enrollment (Table 4 ).

\section{DISCUSSION}

The prevalence of psychological distress in cancer patients varies with the type and stage of cancer, as well as with patient age, gender, and race [7-13]. In a study of 4,496 patients with cancer, the overall prevalence of psychological distress was 35.1\% [12], and other studies have found that $20 \%$ to $47 \%$ of patients who are newly diagnosed or have recurrent cancer show a significant level of distress [13]. In Korea, the prevalence of anxiety or depression in cancer patients was $28.8 \%$ to $56.5 \%$ when all stages and cancer types were considered $[7,8]$. However, there are few studies that have focused on anxiety and depression in patients with gastrointestinal cancer [1416], and no previous study has reported psychological distress in Korean patients with gastrointestinal cancer, despite the incidence of gastrointestinal cancer being relatively high compared with other cancers in Korea [17]. One early study reported that the prevalence of anxiety and depression in newly diagnosed gastrointestinal cancer patients was $17 \%$ and $21 \%$, respectively, and patients with metastatic cancer were more anxious and depressed than patients without metastatic cancer [14]. Another study reported that the prevalence of anxiety and depression in patients with gastrointestinal cancer was $47.2 \%$ and $57 \%$, respectively, and the mean HADS score was 7.6 for the anxiety subscale and 8.4 for the depression subscale [16]. The aim of this study was to assess the prevalence of and the factors associated with anxiety or depression in Korean patients with advanced gastrointestinal cancer. We found that approximately $30 \%$ of patients had anxiety or depression-a statistic consistent with previous studies. Of the 120 study participants, 12 (10.0\%) were classified as having major depressive disorder after assessment by a psychiatrist. This is much higher than has been reported in the general population of South Korea (1.7\% to 2.3\%) [23] and in cancer patients (5\% to $6 \%$ ) [24].

It is important to study predictors of anxiety and/or depression so that clinicians can identify patients that may be more likely to develop these disorders in order to appropriately manage them, allowing for more effective palliation and increased QOL. Previous studies have identified young age, female gender, poor performance status, and certain primary cancer types as predictors of anxiety and depression [7,8,10-12]. In the present study, poor performance status, gastric cancer, and knowledge of advanced cancer were significantly associated with anxiety or depression in patients with advanced gastrointestinal cancer. Performance status had a predictive effect on anxiety or depression, advanced cancer patients with a low performance status are more likely to be depressed or show symptoms of anxiety than those with a high performance status. This finding is in agreement with the findings of other studies $[7,8,10]$. Furthermore, psychological distress may also be affected by primary cancer type [10-12]. Patients with gastric cancer are more vulnerable to psychological distress in connection with the diagnosis than patients with colorectal cancer [14], and Brintzenhofe-Szoc et al. [25] found higher rates of anxiety/depression symptoms in patients with stomach, pancreas, head and neck, or lung cancer. Furthermore, a recent study reported that $31 \%$ of Chinese patients with gastric cancer had depression, and depression was associated with poor survival among gastric cancer patients [26]. Patients with advanced or metastatic gastric cancer commonly experience eating problems caused by dysphagia, anorexia, nausea, vomiting, and abdominal pain, and these problems result in emotional distress. In our study population, knowledge of advanced cancer that could only be treated by palliative therapy affected psychological distress, and patients who knew they had advanced cancer were 15.07 times more likely to be anxious or depressed than those who did not 


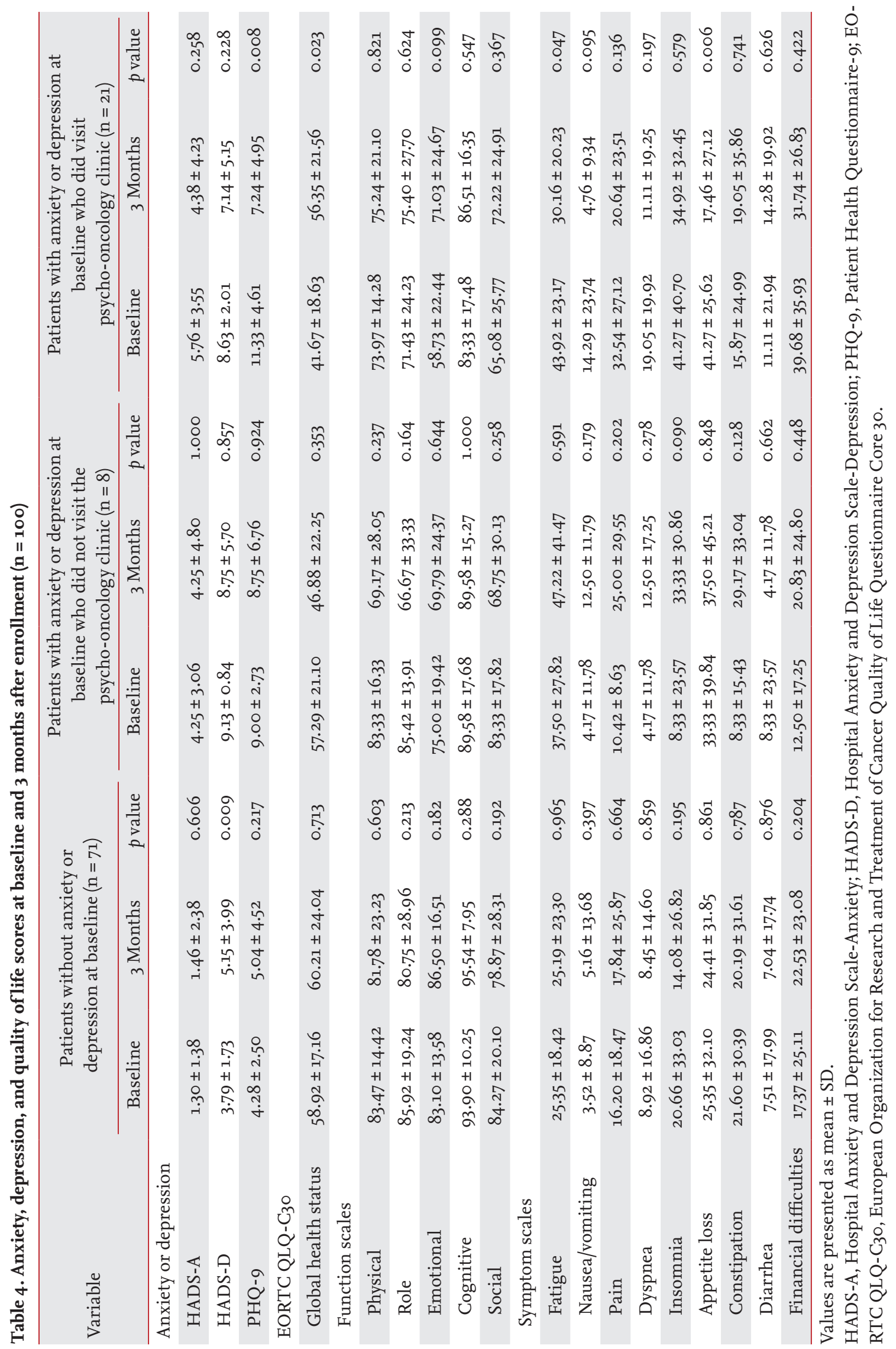



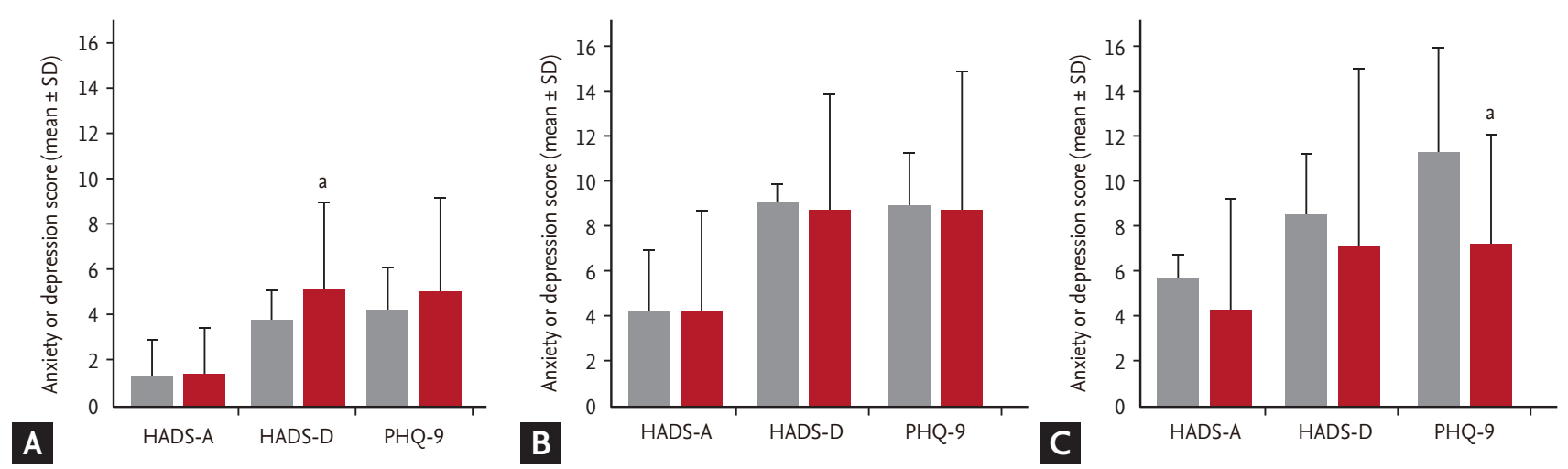

Figure 2. Anxiety and depression at baseline and 3 months after enrollment. (A) Hospital Anxiety and Depression Scale-Anxiety (HADS-A) score, Hospital Anxiety and Depression Scale-Depression (HADS-D) score, and Patient Health Questionnaire-9 (PHQ-9) score in patients without anxiety or depression at baseline, (B) patients with anxiety or depression at baseline who did not visit the psycho-oncology clinic, and (C) patients with anxiety or depression at baseline who did visit the psycho-oncology clinic. Gray bars indicate score at baseline and black bars indicate score at 3 months after enrollment. ${ }^{\mathrm{a}} \mathrm{p}<0.05$.

know of their advanced cancer. Tavoli et al. [16] also reported that patients who knew of their cancer diagnosis had a significantly higher risk of anxiety or depression. Interpretation of a cancer diagnosis as equivalent to death in Middle East and Asian countries may result in emotional distress and concern. For this reason, the patient's family may request that physicians not inform the patient of the diagnosis [9]. However, evidence suggests that sensible disclosure of diagnosis and prognosis is important, and satisfaction with information-giving is associated with a better QOL [27].

There is accumulating evidence of an association between psychological distress and poor outcomes in patients with cancer [4-6]; therefore, treating psychological distress might represent another option for cancer treatment. However, the referral rate from oncologists to psychiatrists is only $4 \%$ to $10 \%$ for cancer patients $[28,29]$. In a study of anxiety and depression disorders in Canadian cancer patients, Wilson et al. [30] found that $60 \%$ of patients with a psychological disorder were not receiving any kind of treatment or counseling. In the current study, we found that patients with anxiety or depression who visited the psycho-oncologic clinic showed an improvement in depression as assessed by PHQ-9 score and in the global health and fatigue dimensions of QOL. Although survival might be considered the ultimate end point in oncology, anxiety or depression is a disabling illness that is associated with poor QOL and therefore warrants intervention, regardless of the potential impact on survival. We suggest that all cancer patients should be screened periodically for psychological symptoms as part of standard cancer care, and patients with psychological problems should be referred to psycho-oncologic clinic for appropriate care.

The present study has several limitations. First, we intended to assess the prevalence of and factors associated with anxiety or depression in patients with advanced gastrointestinal cancer, and the study was not powered to detect the effect of anxiety or depression on survival or any survival benefit resulting from an alleviation of psychological distress. Second, we provided a psychiatric assessment only for patients who were classified as having anxiety or depression according to the HADS or PHQ-9. These instruments are widely used and are a valid measurement of psychological distress in cancer patients. However, we do not have psychiatric diagnoses for those patients who were not identified as having anxiety or depression by the HADS or PHQ-9. Third, the knowledge of advanced cancer at 3 months in patients who did not know they had advanced cancer at baseline may have affected the prevalence of anxiety or depression at 3 months. The prevalence of anxiety or depression at 3 months (four of the 27 patients [13.3\%]) was increased compared with baseline (one of the 30 patients [3.3\%]) in patients who did not know they had advanced cancer at baseline. However, this cannot be accurately assessed because we did not investigate the knowledge of advanced cancer at 3 months. Lastly, the number of patients recruited to the study was small, meaning there was insufficient statistical power for detailed subgroup 
analyses, and all patients were from a single institution, making it difficult to generalize our results.

The results of the present study suggest that the prevalence of anxiety or depression in Korean patients with advanced gastrointestinal cancer is approximately $30 \%$. Physicians should pay attention to the psychological status of patients with metastatic gastrointestinal cancer, particularly those with poor performance status, gastric cancer, or knowledge they had advanced cancer. Furthermore, psychiatric interventions were associated with improvements in depression and QOL in patients with anxiety or depression, suggesting that psychiatric treatment might represent another treatment option for patients with advanced gastrointestinal cancer.

\section{KEY MESSAGE}

1. In this prospective observational study, approximately $30 \%$ of Korean patients with advanced gastrointestinal cancer receiving palliative chemotherapy had anxiety or depression.

2. The risk of anxiety or depression in patients with advanced gastrointestinal cancer was significantly associated with poor performance status, gastric cancer, and knowledge of advanced cancer that could only be treated with palliative therapy.

3. Psychiatric interventions were effective at reducing depression and improving quality of life in patients with anxiety or depression.

\section{Conflict of interest}

No potential conflict of interest relevant to this article was reported.

\section{REFERENCES}

1. Bultz BD, Carlson LE. Emotional distress: the sixth vital sign in cancer care. J Clin Oncol 2005;23:6440-6441.

2. Skarstein J, Aass N, Fossa SD, Skovlund E, Dahl AA. Anxiety and depression in cancer patients: relation between the Hospital Anxiety and Depression Scale and the European Organization for Research and Treatment of Cancer Core Quality of Life Questionnaire. J Psychosom Res
2000;49:27-34.

3. Colleoni M, Mandala M, Peruzzotti G, Robertson C, Bredart A, Goldhirsch A. Depression and degree of acceptance of adjuvant cytotoxic drugs. Lancet 2000;356:13261327.

4. Steel JL, Geller DA, Gamblin TC, Olek MC, Carr BI. Depression, immunity, and survival in patients with hepatobiliary carcinoma. J Clin Oncol 2007;25:2397-2405.

5. Giese-Davis J, Collie K, Rancourt KM, Neri E, Kraemer HC, Spiegel D. Decrease in depression symptoms is associated with longer survival in patients with metastatic breast cancer: a secondary analysis. J Clin Oncol 2011;29:413-420.

6. Pirl WF, Greer JA, Traeger L, et al. Depression and survival in metastatic non-small-cell lung cancer: effects of early palliative care. J Clin Oncol 2012;30:1310-1315.

7. Shim EJ, Shin YW, Jeon HJ, Hahm BJ. Distress and its correlates in Korean cancer patients: pilot use of the distress thermometer and the problem list. Psychooncology 2008;17:548-555.

8. Kim SJ, Rha SY, Song SK, et al. Prevalence and associated factors of psychological distress among Korean cancer patients. Gen Hosp Psychiatry 2011;33:246-252.

9. Jeong A, Shin DW, Kim SY, Yang HK, Park JH. Avoidance of cancer communication, perceived social support, and anxiety and depression among patients with cancer. Psychooncology 2016;25:1301-1307.

10. Salvo N, Zeng L, Zhang L, et al. Frequency of reporting and predictive factors for anxiety and depression in patients with advanced cancer. Clin Oncol (R Coll Radiol) 2012;24:139-148.

11. Linden W, Vodermaier A, Mackenzie R, Greig D. Anxiety and depression after cancer diagnosis: prevalence rates by cancer type, gender, and age. J Affect Disord 2012;141:343351.

12. Zabora J, BrintzenhofeSzoc K, Curbow B, Hooker C, Piantadosi S. The prevalence of psychological distress by cancer site. Psychooncology 2001;10:19-28.

13. Mitchell AJ, Chan M, Bhatti H, et al. Prevalence of depression, anxiety, and adjustment disorder in oncological, haematological, and palliative-care settings: a meta-analysis of 94 interview-based studies. Lancet Oncol 2011;12:160-174.

14. Nordin K, Glimelius B. Psychological reactions in newly diagnosed gastrointestinal cancer patients. Acta Oncol 1997;36:803-810. 
15. Nordin K, Glimelius B. Predicting delayed anxiety and depression in patients with gastrointestinal cancer. $\mathrm{Br} \mathrm{J}$ Cancer 1999;79:525-529.

16. Tavoli A, Mohagheghi MA, Montazeri A, Roshan R, Tavoli Z, Omidvari S. Anxiety and depression in patients with gastrointestinal cancer: does knowledge of cancer diagnosis matter? BMC Gastroenterol 2007;7:28.

17. Jung KW, Won YJ, Kong HJ, et al. Cancer statistics in Korea: incidence, mortality, survival, and prevalence in 2012. Cancer Res Treat 2015;47:127-141.

18. Yoo SW, Kim YS, Noh JS, et al. Validity of Korean version of the mini-international neuropsychiatric interview. Anxiety Mood 2006;2:50-55.

19. American Psychiatric Association. Diagnostic and Statistical Manual of Mental Disorders. 4th ed. Arlington: American Psychiatric Publishing, 2000.

20. Oh SM, Min KJ, Park DB. A study on the standardization of the hospital anxiety and depression scale for Koreans: a comparison of normal, depressed and anxious groups. J Korean Neuropsychiatr Assoc 1999;38:289-296.

21. Han C, Jo SA, Kwak JH, et al. Validation of the Patient Health Questionnaire-9 Korean version in the elderly population: the Ansan Geriatric study. Compr Psychiatry 2008;49:218-223.

22. Yun YH, Park YS, Lee ES, et al. Validation of the Korean version of the EORTC QLQ-C30. Qual Life Res 2004;13:863-868.

23. Cho MJ, Kim JK, Jeon HJ, et al. Lifetime and 12-month prevalence of DSM-IV psychiatric disorders among Korean adults. J Nerv Ment Dis 2007;195:203-210.

24. Derogatis LR, Morrow GR, Fetting J, et al. The prevalence of psychiatric disorders among cancer patients. JAMA 1983;249:751-757.

25. Brintzenhofe-Szoc KM, Levin TT, Li Y, Kissane DW, Zabora JR. Mixed anxiety/depression symptoms in a large cancer cohort: prevalence by cancer type. Psychosomatics 2009;50:383-391.

26. Yu H, Wang Y, Ge X, Wu X, Mao X. Depression and survival in Chinese patients with gastric cancer: a prospective study. Asian Pac J Cancer Prev 2012;13:391-394.

27. Annunziata MA, Foladore S, Magri MD, et al. Does the information level of cancer patients correlate with quality of life? A prospective study. Tumori 1998;84:619-623.

28. Grassi L, Gritti P, Rigatelli M, Gala C. Psychosocial problems secondary to cancer: an Italian multicentre survey of consultation-liaison psychiatry in oncology. Italian Consultation-Liaison Group. Eur J Cancer 2000;36:579585 .

29. McCartney CF, Cahill P, Larson DB, Lyons JS, Wada CY, Pincus HA. Effect of a psychiatric liaison program on consultation rates and on detection of minor psychiatric disorders in cancer patients. Am J Psychiatry 1989;146:898-901.

30. Wilson KG, Chochinov HM, Skirko MG, et al. Depression and anxiety disorders in palliative cancer care. J Pain Symptom Manage 2007;33:118-129. 